\title{
Approximate Entropies of Resting State Continuous Neurocardiovascular Physiological Signals Are Associated with Physical Frailty in Older Adults ${ }^{\dagger}$
}

\author{
Silvin Knight 1,2, Louise Newman ${ }^{1,2}$, John $\mathrm{O}^{\prime}$ Connor 1,2, Rose Anne Kenny 1,2,3 and Roman Romero- \\ Ortuno ${ }^{1,2,3}$ \\ 1 The Irish Longitudinal Study on Ageing (TILDA), Trinity College Dublin, University of Dublin, Dublin, \\ Ireland \\ 2 Discipline of Medical Gerontology, School of Medicine, Trinity College Dublin, University of Dublin, Dublin, \\ Ireland \\ 3 Mercer's Institute for Successful Ageing (MISA), St James's Hospital, Dublin, Ireland \\ † Presented at the Entropy 2021: The Scientific Tool of the 21st Century, 5-7 May 2021; Available online: \\ https://sciforum.net/conference/Entropy2021/.
}

Published: 5 May 2021

Frailty in older adults is characterised by dysregulation in multiple physiological systems. The frailty phenotype is defined on the basis of exhaustion, unexplained weight loss, weakness, slowness and low physical activity (one or two: pre-frail; 3 or more: frail). Our aim was to explore if increasing frailty is associated with the complexity of resting state physiological signals in a large cohort of community-dwelling older adults, enrolled as part of The Irish Longitudinal Study on Ageing (TILDA).

Systolic/diastolic blood pressure (SBP/DBP), mean arterial pressure (MAP), and heart rate (HR) were measured in 3154 participants $\left(66.2 \%\right.$ non-frail; $31.3 \%$ pre-frail; $2.5 \%$ frail) using a Finometer ${ }^{\circledR}$ device at $200 \mathrm{~Hz}$; and frontal lobe oxygenation (tissue saturation index (TSI)) in 2749 individuals (66.3\% non-frail; $31.3 \%$ pre-frail; $2.4 \%$ frail) at $50 \mathrm{~Hz}$ using an Artinis Portalite ${ }^{\circledR}$ near infrared spectroscopy system. Data were acquired continuously during five minutes of supine rest and the last minute (downsampled to $5 \mathrm{~Hz}$ ) was utilised in these analyses. The complexity of signals was quantified using approximate entropy (ApEn) with $m=2$ and an optimal $r$ derived via multiple iterations, implemented in Matlab (R2019a). Statistical analysis was performed using multivariate linear regression models in STATA (v14.1), controlling for age, sex, education, antihypertensive medication, diabetes, number of cardiovascular conditions, smoking, alcohol, and depression.

Mean age for both groups was $64.3 \pm 8.1$ years and $53 \%$ were female. The pre-frail group was associated with significantly increased ApEn for all measures investigated (sBP: $\beta=0.014, p \leq 0.001$; dBP: $\beta=0.009, p=0.002$; MAP: $\beta=0.012, p \leq 0.001$; HR: $\beta=0.011, p=0.003$; TSI: $\beta=0.009, p=0.002$ ). Likewise, the frail group was associated with further increased ApEn for all measures investigated (sBP: $\beta=0.031, p=0.002$; dBP: $\beta=0.028, p=0.003$; MAP: $\beta=0.038, p \leq 0.001$; HR: $\beta=0.034, p=0.001$; TSI: $\beta=$ $0.018, p=0.029)$.

Approximate entropy seems to be a sensitive method to capture increasing signal complexity in multiple physiological systems associated with the frailty phenotype during resting state.

(C) 2021 by the authors. Licensee MDPI, Basel, Switzerland. This article is an open access article distributed under the terms and conditions of the Creative Commons Attribution (CC BY) license (http://creativecommons.org/licenses/by/4.0/). 